\title{
Use of Vanadium Sesquioxide for Electrochemical Supercapacitors
}

\author{
Cameron Wallar, Igor Zhitomirsky \\ McMaster University \\ 1280 Main St. W., Hamilton, Canada \\ wallarcj@mcmaster.ca
}

\section{Extended Abstract}

Electrochemical supercapacitors are unique energy storage devices that could potentially find synergistic or standalone application in a variety of products and fields such as electric vehicles, portable electronics and renewable energy. The high theoretical energy densities of materials that display faradaic pseudo-capacitance are of particular interest. A number of transition metal oxides are known to display faradaic pseudo-capacitance such as $\mathrm{RuO}_{2}, \mathrm{MnO}_{2}$ and $\mathrm{V}_{2} \mathrm{O}_{5}$. With the exception of $\mathrm{RuO}_{2}$, most pseudo-capacitive transition metal oxides are intrinsically poor electrical conductors, which can limit their performance under high power conditions. This is often combated by mixing the active material with conductive additives, however the low intrinsic conductivity of the oxide is still a performance limiter. The problem is further exasperated when the high mass loadings necessary for practical energy densities are used. The success of supercapacitor technology will depend largely on the ability to utilize the high capacitance of advanced charge storage materials in electrodes with high active mass loading. The objective of this research was to investigate the use of vanadium sesquioxide $\left(\mathrm{V}_{2} \mathrm{O}_{3}\right)$ as an active material for such a supercapacitor electrode.

$\mathrm{V}_{2} \mathrm{O}_{3}$ transforms from an antiferromagnetic insulating phase to a paramagnetic metallic phase at $\sim 160 * \mathrm{~K}$. As outlined above, this property makes it a potentially promising material for high power pseudo-capacitors. $\mathrm{A}_{2} \mathrm{O}_{3}$-carbon nanotube composite was produced through the use of a chelating co-dispersant, which allowed for strong adsorption, co-dispersion and mixing of both components. This composite was then impregnated into high porosity nickel foam current collectors up to a mass loading of $\sim 30 \mathrm{mg} / \mathrm{cm}^{2}$ and tested in aqueous $0.5 \mathrm{M} \mathrm{Na}_{2} \mathrm{SO}_{4}$ using a three-electrode setup. A saturated calomel electrode (SCE) was used as the reference electrode and platinum gauze as the counter electrode. Electrodes were characterized using cyclic voltammetry and impedance spectroscopy. The composite material was investigated using XPS, XRD and SEM before and after testing. The electrodes exhibited virtually ideal capacitive behaviour in the voltage window 0-0.7 V vs SCE after an initial electrochemical activation process. The results of XPS, XRD and SEM studies of the active material before and after testing provided an insight into the charge storage mechanism and microstructure changes during cycling. An enrichment of $\mathrm{V}^{5+}$ was observed on the surface while the bulk crystal structure remained intact. Cyclic voltammetry and impedance spectroscopy data showed that a capacitance above $4 \mathrm{~F} \mathrm{~cm}^{-2}$ can be achieved at low scan rates. The electrodes showed low impedance and excellent cyclic stability, with no degradation in capacitance after 1000 cycles. Testing results indicate that $\mathrm{V}_{2} \mathrm{O}_{3}$ is a promising material for advanced supercapacitors with high active mass, high active material to current collector mass ratio, low impedance and high power-energy characteristics. 